\title{
Effects of Apatinib on the "Stemness" of Non-Small-Cell Lung Cancer Cells In Vivo and Its Related Mechanisms
}

\author{
Bin Yang, ${ }^{1}$ Yan Wang, ${ }^{2}$ Zhuoying Chen $\mathbb{D},{ }^{3}$ Yi-Ming Feng, ${ }^{4}$ and Liang-Liang Shi ${ }^{5}$ \\ ${ }^{1}$ Department of Oncology, Hubei Cancer Hospital, Tongji Medical College, Huazhong University of Science and Technology, \\ Wuhan 430079, China \\ ${ }^{2}$ Department of Oncology, Liyuan Hospital, Tongji Medical College, Huazhong University of Science and Technology, \\ Wuhan 430077, China \\ ${ }^{3}$ Department of Geriatrics, Liyuan Hospital, Tongji Medical College, Huazhong University of Science and Technology, \\ Wuhan 430077, China \\ ${ }^{4}$ Department of Radiology, Union Hospital, Tongji Medical College, Huazhong University of Science and Technology, \\ Wuhan 430022, China \\ ${ }^{5}$ Cancer Center, Union Hospital, Tongji Medical College, Huazhong University of Science and Technology, Wuhan 430022, China
}

Correspondence should be addressed to Liang-Liang Shi; sllhust@hust.edu.cn

Received 11 March 2020; Revised 29 May 2020; Accepted 10 June 2020; Published 11 August 2020

Academic Editor: Rafael Sirera

Copyright (c) 2020 Bin Yang et al. This is an open access article distributed under the Creative Commons Attribution License, which permits unrestricted use, distribution, and reproduction in any medium, provided the original work is properly cited.

Objective. To investigate the effects of Apatinib on the "stemness" of lung cancer cells in vivo and to explore its related mechanisms. Methods. A xenograft model of lung cancer cells A549 was established in nude mice and randomized into a control group $(n=4)$ and an Apatinib group $(n=4)$. Tumor tissues were harvested after 2 weeks, and mRNA was extracted to detect changes in stemness-related genes (CD133, EPCAM, CD13, CD90, ALDH1, CD44, CD45, SOX2, NANOG, and OCT4) and Wnt/ $\beta$-catenin, Hedgehog, and Hippo signal pathways. Results. Compared with the control group, the volume and weight of nude mice treated with Apatinib were different and had statistical significance. Apatinib inhibited the expressions of ABCG2, CD24, ICAM-1, OCT4, and SOX2 and upregulated the expressions of CD44, CD13, and FOXD3. Apatinib treatment also inhibited the Wnt/ $\beta$-catenin, Hedgehog, and Hippo signaling pathways. Conclusion. Apatinib suppressed the growth of non-small-cell lung cancer cells by repressing the stemness of lung cancer through the inhibition of the Hedgehog, Hippo, and Wnt signaling pathways.

\section{Introduction}

Lung cancer is one of the most common malignant tumors in the world. Approximately 1.8 million people are diagnosed with lung cancer every year, with 1.6 million people dying from the deadly disease [1]. The 5 -year survival rate for lung cancer is still less than $20 \%$, although considerable progress has been made in various treatments, such as surgery, radiotherapy, chemotherapy, and targeted therapy. Targeted therapy is one of the essential treatment methods for advanced non-small-cell lung cancer [2]. At present, epidermal growth factor receptor (EGFR) tyrosine kinase inhibitors (TKI), anaplastic lymphoma kinase $(A L K)$ tyrosine kinase inhibitors and ROS1 inhibitors, epidermal growth factor receptor $(E G F R)$ inhibitors, mesenchymal-epithelial transition factor (MET) inhibitors, epidermal growth factor receptor 2 (EGFR) inhibitors, murine sarcoma virus oncogene congener B1 (B1BRAF) inhibitors, Kristen murine sarcoma virus oncogene homologues (KRAS) inhibitors, and other targeted drugs are emerging. Targeted drugs have significantly improved and prolonged the overall survival (OS) and progression-free survival (PFS) of patients [3]. However, each targeted drug is only suitable for lung cancer patients with specific genetic mutations, and since many patients do not have the above known genetic mutations, they cannot receive targeted therapy and, thus, have a short survival time.

Apatinib is an antiangiogenic drug developed independently in China. Apatinib has been used before for the second-line treatment of gastric cancer. Studies have shown that Apatinib can also be used in the treatment of liver cancer after chemotherapy has failed [4]. The main antitumor mechanism of 
TABle 1: Characteristics of the primers used for real-time PCR.

\begin{tabular}{|c|c|c|}
\hline Genes & Primers (forward and reverse) & $\begin{array}{l}\text { Products } \\
\text { (bp) }\end{array}$ \\
\hline PTCH & $\begin{array}{c}\text { 5'-TGGAACGAGGACAAAGCGG-3' }^{\prime} \\
5^{\prime} \text {-AGGCATAGGCGAGCATGAGTAA-3' }\end{array}$ & 202 \\
\hline SMO & $\begin{array}{l}5^{\prime} \text {-TCTCGGGCAAGACCTCCTACT-3' } \\
5^{\prime} \text {-CGCACGGTATCGGTAGTTCTT-3' }\end{array}$ & 146 \\
\hline GLI1 & $\begin{array}{l}5^{\prime} \text {-CCAAGCACCAGAATCGGACC-3' } \\
5^{\prime} \text {-TTTGGTCACATGGGCGTCAG-3' }\end{array}$ & 140 \\
\hline GLI2 & $\begin{array}{l}5^{\prime} \text {-AGGGATGACTGTAAGCAGGAGG- }{ }^{\prime} \\
5^{\prime} \text {-CGGCACACAAACTCCTTCTTCT- } 3^{\prime}\end{array}$ & 152 \\
\hline GLI3 & $\begin{array}{c}5^{\prime} \text {-GCACTAAGCGTTACACAGACCCA-3' } \\
5^{\prime} \text {-CTTCTCTGCCTTGACGGTTTTC- } 3^{\prime}\end{array}$ & 257 \\
\hline $\mathrm{SHH}$ & $\begin{array}{c}5^{\prime} \text {-CTGCTGGTATGCTCGGGACT-3' } \\
5^{\prime} \text {-CATTGGGGATAAACTGCTTGTAG-3 }{ }^{\prime}\end{array}$ & 106 \\
\hline DHH & $\begin{array}{c}\text { 5'-GATGACCGAGCGTTGTAAGGA-3' }^{\prime} \\
5^{\prime} \text {-GTTATCAGCTTTGACCGACACG }\end{array}$ & 277 \\
\hline FU & $\begin{array}{l}5^{\prime} \text {-TTGCTGCCCAGTTGGTGTCA-3' } \\
\text { 5'-TGTGGTCGTATGGTCGCTCCT-3' }^{\prime} \text {-TGTCG }\end{array}$ & 225 \\
\hline GAS1 & $\begin{array}{l}5^{\prime} \text {-ATCTGCGAGTCGGTCAAGGA-3' } \\
5^{\prime} \text {-TGCGCTGCTCGTCATCGTA-3' }\end{array}$ & 133 \\
\hline Cdon & $\begin{array}{l}5^{\prime} \text {-CTTTTCCAGCCGTCCAATAACT-3' } \\
5^{\prime} \text {-GTGCCACTGCTTTGAACCTTCT-3' }\end{array}$ & 205 \\
\hline BOC & $\begin{array}{l}5^{\prime} \text {-CATCACTGCCCTTAACAACCAC-3' } \\
5^{\prime} \text {-TGTTTCCCTCATCCACTTCAATC- } 3^{\prime}\end{array}$ & 158 \\
\hline $\mathrm{HH}$ & $\begin{array}{l}\text { 5'-TCACCGTCTGGCACCCTAGT-3' } \\
5^{\prime} \text {-CATGGCTCCTCTTGAACCCTG-3' }\end{array}$ & 120 \\
\hline HHIP & $\begin{array}{l}5^{\prime} \text {-GAGAAGGTGCCTGAATGGGAAC-3' } \\
5^{\prime} \text {-GGTGAGTGGAACAGGCTTTGA-3' }\end{array}$ & 270 \\
\hline SUFU & $\begin{array}{c}\text { 5'-GCCTTCGCTTCGCTCTTTC-3' } \\
\text { 5'-AGGCCGAAGCTGATGTAGTGC-3' }\end{array}$ & 212 \\
\hline MST1 & $\begin{array}{l}\text { 5'-CGGGTCCCAGTAGCCAAGAT-3' } \\
\text { 5'-GTGTCATTACCCGTACCTTTGG-3' }^{\prime}\end{array}$ & 191 \\
\hline MST2 & $\begin{array}{l}5^{\prime} \text {-ACCATCTGCCTTAGGAACGGA-3' } \\
5^{\prime} \text {-TAATTGCGACAACTTGACCGG-3' }\end{array}$ & 223 \\
\hline LATS1 & $\begin{array}{l}5^{\prime} \text {-CCTATTAATGCCAGCATGAAACC- } 3^{\prime} \\
5^{\prime} \text {-CGTTGCTAGGGTGAGCTTGA-3' }\end{array}$ & 220 \\
\hline LATS & $\begin{array}{l}5^{\prime} \text {-GAGCAGATTGTGCGGGTCATT-3' } \\
5^{\prime} \text {-TGGTGGTAGGACGCAAACGA-3' }\end{array}$ & 116 \\
\hline SAV1 & $\begin{array}{l}5^{\prime} \text {-GAATGCCACAGAATCAGGGGA-3' } \\
5^{\prime} \text {-GATGCCTGTATTGGGCCTTCTT-3' }\end{array}$ & 291 \\
\hline MOB1A & $\begin{array}{l}5^{\prime} \text {-AGTGGGAATCTGAGACAAGCTG-3' } \\
5^{\prime} \text {-GGTGCAGAACATTTGATTGGCT- } 3^{\prime}\end{array}$ & 224 \\
\hline MOB1B & $\begin{array}{c}5^{\prime} \text {-TGGGCAGATGGAACGAACA-3' } \\
\text { 5'-GAAGCTGGATCACAGGGTCAA-3 }^{\prime}\end{array}$ & 232 \\
\hline YAP & $\begin{array}{l}\text { 5'-GAGTTAGCCCTGCGTAGCCA-3' }^{\prime} \\
5^{\prime} \text {-GGCAGGGTGCTTTGGTTGATA-3' }\end{array}$ & 272 \\
\hline TAZ & 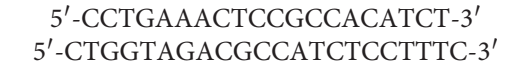 & 235 \\
\hline Notch1 & $\begin{array}{l}\text { 5'-GTCAACGCCGTAGATGACC-3' } \\
5^{\prime} \text {-TTGTTAGCCCCGTTCTTCAG-3' }\end{array}$ & 101 \\
\hline Notch2 & $\begin{array}{c}5^{\prime} \text {-ACTGTGAGGAGCAACTCGAT-3' } \\
5^{\prime} \text {-TCCACTTCATACTCACAGTTGA-3' }\end{array}$ & 133 \\
\hline Notch3 & $\begin{array}{l}5^{\prime} \text {-TGACCGTACTGGCGAGACT-3' } \\
5^{\prime} \text {-CCGCTTGGCTGCATCAGCA-3' }\end{array}$ & 67 \\
\hline Notch4 & $\begin{array}{l}5^{\prime} \text {-AACTCCTCCCCAGGAATCTG-3' } \\
5^{\prime} \text {-CCTCCATCCAGCAGAGGTT-3' }\end{array}$ & 168 \\
\hline JAG1 & $\begin{array}{l}5^{\prime} \text {-GCCAGGAAGTTTCAGGGAGA-3' } \\
5^{\prime} \text {-GCTGGAGACTGGAAGACCGA-3' }\end{array}$ & 264 \\
\hline JAG2 & $\begin{array}{l}5^{\prime} \text {-CTGACTGCCGCATCAACATC-3' } \\
5^{\prime} \text {-GCTACAGCGATACCCGTTGA-3' }\end{array}$ & 92 \\
\hline DLL1 & $\begin{array}{l}5^{\prime}-\text { TCTCCTGATGACCTCGCAACA-3' } \\
5^{\prime} \text {-TCACACACGAAGCGGTAGGAG-3' }\end{array}$ & 149 \\
\hline DLL3 & $\begin{array}{l}5^{\prime} \text {-CACTCAACAACCTAAGGACGCA-3' } \\
5^{\prime} \text {-CGAGGAAGGGTAGGGAAAAAG-3' }\end{array}$ & 212 \\
\hline DLL4 & $\begin{array}{l}\text { 5'-GACCTCTCCACAGACACCTTTG-3' } \\
5^{\prime} \text {-TCCACTTCCAGCTCCTTCTTCT-3' }\end{array}$ & 299 \\
\hline HES1 & $\begin{array}{c}5^{\prime} \text {-ATTCTGGAAATGACAGTGAAGCAC-3' } \\
5^{\prime} \text {-CACCTCGGTATTAACGCCCTC-3' }\end{array}$ & 168 \\
\hline
\end{tabular}

Table 1: Continued.

\begin{tabular}{|c|c|c|}
\hline Genes & Primers (forward and reverse) & $\begin{array}{l}\text { Products } \\
\quad \text { (bp) }\end{array}$ \\
\hline HES5 & $\begin{array}{l}\text { 5'-GAAAAACCGACTGCGGAAGC-3' }^{\prime} \\
5^{\prime} \text {-GACGAAGGCTTTGCTGTGCT-3' }\end{array}$ & 184 \\
\hline NGN & $\begin{array}{l}5^{\prime} \text {-CAGATTTGGTCCCATTTGTGAG-3' } \\
5^{\prime} \text {-ATGGCAACACTACATCCTGACC-3' }\end{array}$ & 279 \\
\hline Heyl & $\begin{array}{l}5^{\prime} \text {-GAAGCAGGTAATGGAGCAAGGA-3' } \\
5^{\prime} \text {-GAAGCGTAGTTGTTGAGATGCG-3' }\end{array}$ & 258 \\
\hline HEY2 & $\begin{array}{l}5^{\prime} \text {-AAGGCTACTTTGACGCACACG-3' } \\
5^{\prime} \text {-GAGATGAGACACAAGCCGCAC-3' }\end{array}$ & 146 \\
\hline HES1 & $\begin{array}{l}5^{\prime} \text {-ATTCTGGAAATGACAGTGAAGCAC- } 3^{\prime} \\
5^{\prime} \text {-CACCTCGGTATTAACGCCCTC- } 3^{\prime}\end{array}$ & 168 \\
\hline DV1 & $\begin{array}{l}\text { 5'-AGAAGTCAGCTCTTGCCTCAGTT-3' } \\
5^{\prime} \text {-ATCTCATCAGTAGCACGACGAAG-3' }\end{array}$ & 256 \\
\hline AXIN & $\begin{array}{c}5^{\prime} \text {-TCTGGATACCTGCCGACCTTA-3' } \\
5^{\prime} \text {-TCTGCTGCTCGCTGTCGTT-3' }\end{array}$ & 271 \\
\hline APC & $\begin{array}{l}5^{\prime} \text {-CAAAACTGGAAACTGAGGCATCT-3' } \\
5^{\prime} \text {-ACTCTCCAGAACGGCTTGATACA-3 } 3^{\prime}\end{array}$ & 225 \\
\hline$\beta$-catenin & $\begin{array}{l}5^{\prime} \text {-GCCAAGTGGGTGGTATAGAGG-3' } \\
5^{\prime} \text {-GGGATGGTGGGTGTAAGAGC-3' }\end{array}$ & 192 \\
\hline GSK3B & $\begin{array}{l}\text { 5'-GTTAGCAGAGACAAGGACGGCA-3' }^{\prime} \\
5^{\prime} \text {-GCAATACTTTCTTGATGGCGAC-3' }\end{array}$ & 183 \\
\hline GBP & $\begin{array}{c}\text { 5'-AGGTGGCTCCTGACGCTAA-3' } \\
\text { 5'-CAGGCTGGAAGGGAAAGACA-3' }^{\prime}\end{array}$ & 180 \\
\hline TCF & $\begin{array}{l}5^{\prime} \text {-GAGTATGCCTACCTCAAAGCCA-3' } \\
5^{\prime} \text {-AGCCGCTTGATCTTCCCTG-3' }\end{array}$ & 80 \\
\hline FRZB & $\begin{array}{c}5^{\prime} \text {-ACGGAAACTGTAGAGGGGCA-3' } \\
5^{\prime} \text {-CAGTGTCCCGTGGAATGTTTAC- }{ }^{\prime}\end{array}$ & 204 \\
\hline $\mathrm{c}-\mathrm{Myc}$ & $\begin{array}{l}\text { 5'-TGCTGCCAAGAGGGTCAAGT-3' } \\
5^{\prime} \text {-GCTCCGTTTTAGCTCGTTCC-3' }\end{array}$ & 160 \\
\hline PKC & $\begin{array}{l}5^{\prime} \text {-GCTTATGCTGTCATGTCCCGG-3' } \\
5^{\prime} \text {-GATGATGAGGACTCCCCCCA-3' }\end{array}$ & 120 \\
\hline RHOA & $\begin{array}{l}5^{\prime} \text {-ATTGTTGGTGATGGAGCCTGTG-3' } \\
5^{\prime} \text {-GTGGGCACATACACCTCTGG-3' }\end{array}$ & 83 \\
\hline ILK & $\begin{array}{c}\text { 5'-GCAGTGAATGAACACGGGAAT-3' }^{\prime} \\
5^{\prime} \text {-CACCAGGTCCTCTGCCACTT- } 3^{\prime}\end{array}$ & 78 \\
\hline $\mathrm{CD} 24$ & $\begin{array}{l}\text { 5'-GCTCCTACCCACGCAGATTT-3' } \\
\text { 5'-GGTGGCATTAGTTGGATTTGG-3' }^{\prime}\end{array}$ & 109 \\
\hline $\mathrm{CD} 13$ & $\begin{array}{c}5^{\prime} \text {-CCGACATTGACAAGACTGAGCT- } 3^{\prime} \\
5^{\prime} \text {-ACCTTTCTGACATTGCCCTCC- } 3^{\prime}\end{array}$ & 175 \\
\hline $\mathrm{CD} 44$ & $\begin{array}{l}5^{\prime} \text {-TCCAACACCTCCCAGTATGACA-3' } \\
5^{\prime} \text {-CTTTCTGGACATAGCGGGTG-3' }\end{array}$ & 151 \\
\hline $\mathrm{CD} 90$ & $\begin{array}{l}5^{\prime} \text {-CGCTCTCCTGCTAACAGTCTTG- }{ }^{\prime} \\
5^{\prime} \text {-GTTCGGGAGCGGTATGTGTG-3' }\end{array}$ & 213 \\
\hline CD45 & $\begin{array}{l}5^{\prime} \text {-TGAGGAGCAAGGAAGCCAATC- } 3^{\prime} \\
5^{\prime} \text {-GCCACCAACTGAAGGCTGAAC- } 3^{\prime}\end{array}$ & 77 \\
\hline ALDH1 & $\begin{array}{c}5^{\prime} \text {-TGCCGACTTGGACAATGCT- } 3^{\prime} \\
5^{\prime} \text {-GCCTCCTCCACATTCCAGTTT-3 }\end{array}$ & 277 \\
\hline ICAM1 & $\begin{array}{l}5^{\prime} \text {-CCGTTGCCTAAAAAGGAGTTGC-3' } \\
5^{\prime} \text {-TGGCAGCGTAGGGTAAGGTTC- } 3^{\prime}\end{array}$ & 221 \\
\hline LGR5 & $\begin{array}{l}5^{\prime} \text {-CGGGAAACGCTCTGACATACAT-3' } \\
5^{\prime} \text {-ACTTCTAAAAGCCTGGACGGG-3' }\end{array}$ & 260 \\
\hline CK7 & $\begin{array}{l}5^{\prime} \text {-TGAAGAAGGATGTGGATGCTGC-3' } \\
5^{\prime} \text {-CAGCTCTGTCAACTCCGTCTCAT-3' }\end{array}$ & 119 \\
\hline CK19 & $\begin{array}{l}5^{\prime} \text {-AGAATTGAACCGGGAGGTCG-3' } \\
5^{\prime} \text {-CCTGATTCTGCCGCTCACTA-3' }\end{array}$ & 257 \\
\hline CD34 & $\begin{array}{l}5^{\prime} \text {-GCAACATCTCCCACTAAACCCTA-3' } \\
5^{\prime} \text {-GTCCTTCTTAAACTCCGCACAGC-3' }\end{array}$ & 156 \\
\hline $\mathrm{CD} 47$ & $\begin{array}{l}5^{\prime} \text {-ACCTCCTTCGTCATTGCCATATT-3' } \\
5^{\prime} \text {-ATACACGCCGCAATACAGAGAC- } 3^{\prime}\end{array}$ & 89 \\
\hline DLK1 & $\begin{array}{c}5^{\prime} \text {-CCCTTTGTGACCAGTGCGTG-3' } \\
\text { 5'-ATTCATAGAGGCCATCGTCCAG-3' }^{\prime}\end{array}$ & 183 \\
\hline EpCAM & $\begin{array}{c}\text { 5'-TAATCGTCAATGCCAGTGTACTTC-3' } \\
\text { 5'-AGCCATTCATTTCTGCCTTCAT-3' }\end{array}$ & 101 \\
\hline CD133 & $\begin{array}{l}5^{\prime} \text {-TGGCATCTTCTATGGTTTTGTGG-3' } \\
5^{\prime} \text {-TCCTTGGTAGTGTTGTACTGGGC-3' }\end{array}$ & 159 \\
\hline OCT4 & $\begin{array}{l}5^{\prime} \text {-GAGTGAGAGGCAACCTGGAGAAT-3' } \\
5^{\prime} \text {-ACCGAGGAGTACAGTGCAGTGAA-3' }\end{array}$ & 292 \\
\hline
\end{tabular}


TABle 1: Continued.

\begin{tabular}{|c|c|c|}
\hline Genes & Primers (forward and reverse) & $\begin{array}{l}\text { Products } \\
\text { (bp) }\end{array}$ \\
\hline SOX2 & $\begin{array}{c}\text { 5'-GGTTACCTCTTCCTCCCACTCC-3' }^{\prime} \\
5^{\prime} \text {-CGCTCTGGTAGTGCTGGGAC-3' }\end{array}$ & 140 \\
\hline NANOG & $\begin{array}{l}5^{\prime} \text {-GAGAAGAGTGTCGCAAAAAAGGA-3' } \\
5^{\prime} \text {-TGAGGTTCAGGATGTTGGAGAGT- } 3^{\prime}\end{array}$ & 163 \\
\hline FOXD3 & $\begin{array}{l}5^{\prime} \text {-GCAACTACTGGACCCTGGAC-3' } \\
5^{\prime} \text {-TAAGCGCCGAAGCTCTGCAT-3' }\end{array}$ & 142 \\
\hline SALL4 & $\begin{array}{c}5^{\prime} \text {-CATCAACTCGGAGGAGGACCA-3' } \\
5^{\prime} \text {-TGATGAGGACAGGTGGATTTTTAGT-3' }\end{array}$ & 275 \\
\hline$\beta$-actin & $\begin{array}{c}5^{\prime} \text {-AGTTGCGTTACACCCTTTCTTGAC-3' } \\
5^{\prime} \text {-GCTCGCTCCAACCGACTGC-3' }\end{array}$ & 171 \\
\hline
\end{tabular}

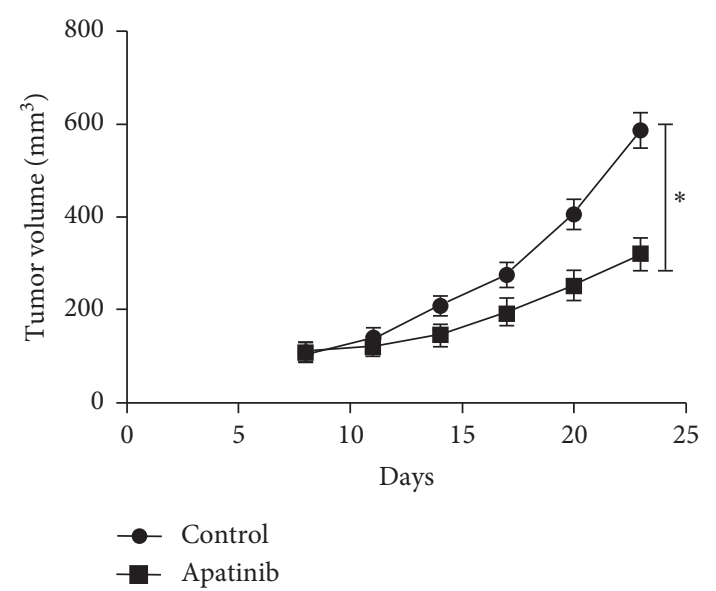

(a)

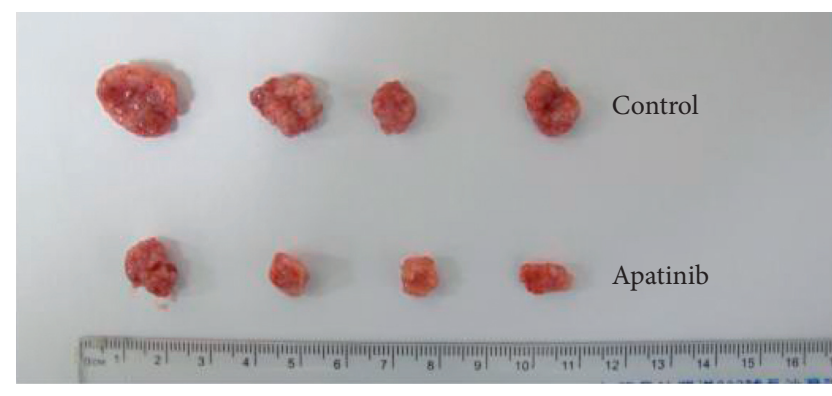

(b)

FIGURE 1: Effect of Apatinib on the volume change of subcutaneous transplanted tumor in nude mice, ${ }^{*} P<0.05$.

the drug is to inhibit tumor growth by inhibiting vascular endothelial growth factor 2 (VEGFR-2) and then inhibiting tumor angiogenesis [5]. Previous investigations have indicated that antiangiogenic drugs can inhibit the growth of multiple types of solid tumors, including non-small-cell lung cancer (NSCLC) [6]. Wang et al. included 128 patients with NSCLC with the failure of first-line treatment. Their results showed that patients who received a combined therapy of Apatinib and chemotherapy had significantly prolonged PFS and OS compared to those who underwent chemotherapy alone [7].

Lung cancer stem-like cells belong to a subpopulation of undifferentiated lung tumor cells that possess the ability to proliferate indefinitely and renew themselves, which is critical for lung cancer tumorigenesis, metastasis, resistance to therapy, and disease relapse [8]. Some studies have shown that lung cancer stem cells are the origin of malignant manifestations of lung cancer. Lung cancer stem cells can promote tumor proliferation through Hedgehog, Wnt, Hippo, and other signaling pathways. Moreover, lung cancer stem cells express "stemness"-related biomarkers, such as CD44, CD24, CA133, CD13, CD90, ABCG2, SOX2, and EPCAM [9]. Therefore, new therapeutic strategies targeting these signaling pathways could contribute to the regulation of stem cell replication, survival, and differentiation.

In this study, we established a subcutaneous NSCLC xenotransplantation in nude mice to observe the effects of Apatinib on the expression of surface markers of lung cancer stem cells, totipotent factors, and stemness-related genes and signal pathways, providing theoretical bases for drug selection of advanced non-small-cell lung cancer.

\section{Materials and Methods}

2.1. Animals and NSCLC Xenotransplantation. We purchased four-to-five-week-old female and male BALB/c nude mice from Vital River Laboratory Animal Technology Co. Ltd. (Beijing, China). All animal experiments were carried out in line with the National Institutes of Health guide for the care and use of laboratory animals. Approximately $1 \times 10^{7}$ A549 cells were suspended in $1 \mathrm{ml}$ phosphate-buffered solution (PBS), and the cell suspension $(0.2 \mathrm{~mL})$ was injected subcutaneously into the right axillary of nude mice and then reared in a cage under SPF condition.

After the inoculation of all nude mice, the general condition and tumorigenesis of the inoculation sites were observed and recorded every day. Eight nude mice (7 days after tumor inoculation) were randomized into a control group $(n=4)$ and an Apatinib group $(n=4)$. The two groups of mice were subjected to treatment by oral gavage once daily for 14 consecutive days, as follows: (a) Apatinib (50 mg/kg) (Hengrui Pharmaceutical company, Jiangsu, China); (b) DMSO $(100 \mu \mathrm{L} /$ $20 \mathrm{~g}$ ) (MP Biomedicals, USA); the maximum diameter (A) and the minimum diameter (B) of the tumors were measured with calipers on the $1^{\text {st }}, 5^{\text {th }}, 8^{\text {th }}, 11^{\text {th }}$, and $14^{\text {th }}$ day after the drug administration and 24 hours after the end of the gavage, and the tumor volume, $(V)=\left(A \times B^{2}\right) / 2$, was calculated. The tumor tissues from the A549 xenografts were separated and collected after the last drug administration on day 14. These tumor tissues were frozen immediately in liquid nitrogen and stored for qPCR analysis. The growth curves of the xenotransplantation tumors of the experimental and control groups were plotted using GraphPad Prism 7.

2.2. Detecting the Expression Level of "Stemness"-Related Genes in Tumor Tissues by Real-Time PCR. $100 \mathrm{mg}$ of subcutaneous xenograft tissues from each of the Apatinib group and the control group was used for this investigation. The total RNA was extracted using RNA extraction reagent Trizol (Life Technologies, Grand Island, NY, USA) following the manufacturer's instructions. Reverse transcription was performed with PrimeScript RT Reagent Kit (Vazyme, Thermo Fisher, NY, USA). qRT-PCR was carried out with the SYBR ${ }^{\circledR}$ Green PCR Master Mix (Life Technologies, Grand Island, NY, USA) in conformity with the 

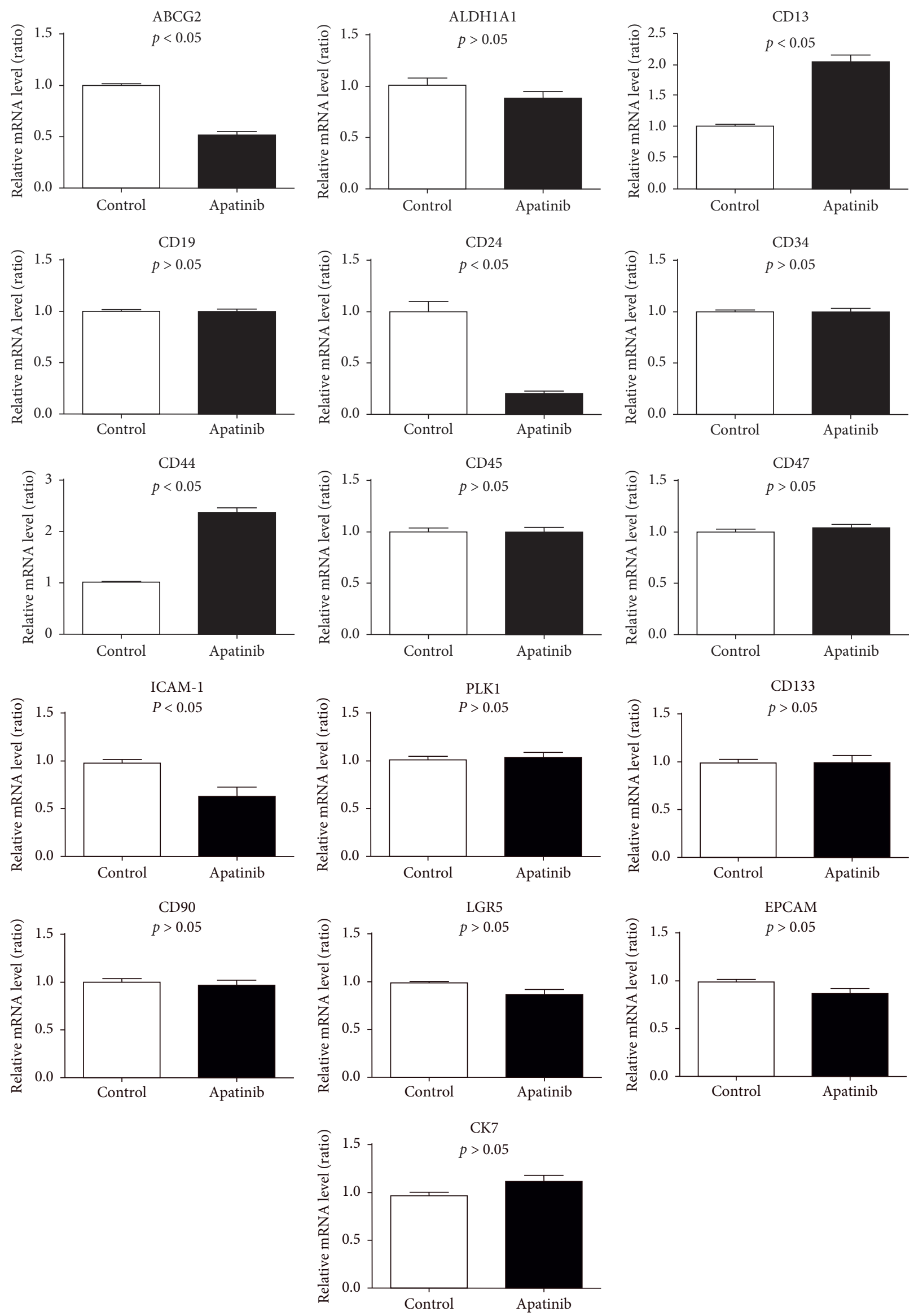

Figure 2: Effect of Apatinib on the surface markers of lung cancer stem cells. 

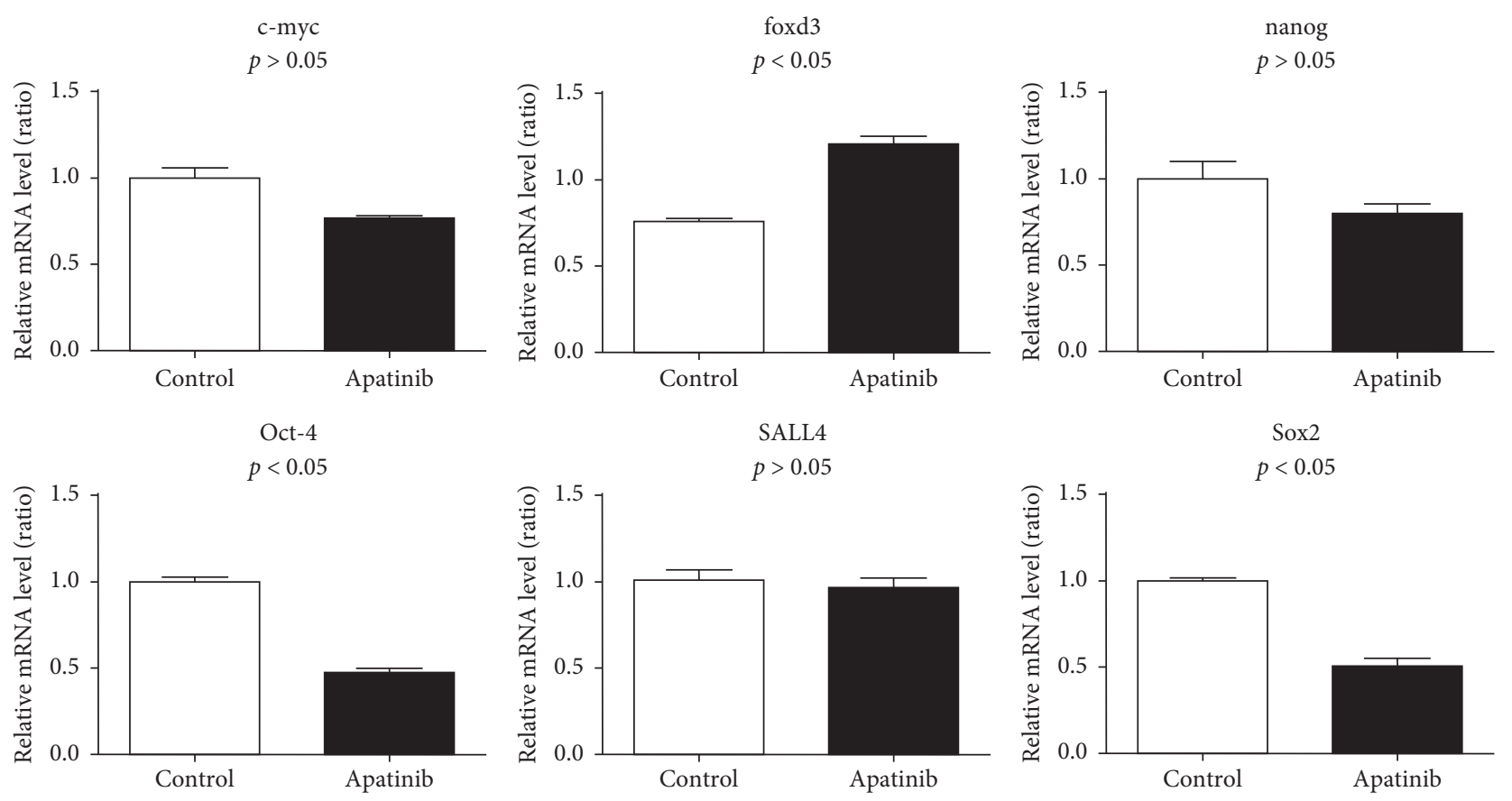

Figure 3: Effect of Apatinib on versatile regulators of lung cancer stem cells.

manufacturer's instructions. The sequences for sense $(S)$ and antisense (A) primers are shown in Table 1.

2.3. Statistical Analysis. The SPSS 22.0 statistical software (SPSS Inc., Chicago, IL, USA) was used for the analysis of statistical data. Data are expressed as mean \pm standard deviation $(x \pm s)$. Two-group comparisons were made using the independent-samples $t$-test, while multiple group comparisons were conducted using the least significant difference test. Values of $P<0.05$ were considered statistically significant.

\section{Results}

3.1. Comparison of Growth of Subcutaneous Xenografts between Apatinib and Control Groups. Drugs were administered when the tumor volume of the nude mice in the two groups reached about $100 \mathrm{~mm}^{3}$. The body weight and tumor volume were measured and recorded on days $8,11,14,17$, and 20 after inoculation; the volume change curve is shown in Figure 1(a). The results indicated that the growth and volume of tumors were slower and smaller, respectively, in the Apatinib group than in the control group $(P<0.05)$. The final tumor masses of the two groups were compared as shown below (Figure 1(b)).

\subsection{Expression of Stemness-Related Genes in NSCLC Subcu-} taneous Xenografts in Nude Mice at mRNA Level. According to the real-time PCR detection results, for the surface marker-related genes of lung cancer stem cells, the expressions of CD13 and CD44 were upregulated in the Apatinib group when compared to the control group, whereas the expressions of $A B C G 2, C D 24$, and ICAM-1 were downregulated. There were no significant differences in the expressions of DLK1, CD133, CD90, CD45, ALDH1, LGR5, CD34, CD47, EPCAM, CK7, and CK19 between both groups (Figure 2).

For the totipotent factor, FOXD3 expression was elevated in the Apatinib-treated group, whereas OCT4 and SOX2 expressions were downregulated, and the expressions of C-MYC, NANOG, and SALL4 showed no remarkable differences when compared to the control group (Figure 3 ).

For the Hedgehog signaling pathway-related genes, the expression of HHIP apparently increased in the Apatinib treatment group, while the expressions of $H H, S H H$, and $F U$ were downregulated, but there were no significant differences between PTCH, SMO, GLI3, GLI1, GLI2, DHH, GAS1, CDO, $B O C$, and SUFU expressions between both groups (Figure 4).

For the Hippo signaling pathway-related genes, the expressions of LAST1 and LAST2 were downregulated in the Apatinib-treated group, but there were no significant differences in the expressions of MST2, MST1, SAV1, MOB1A, $M O B 1 B, Y A P$, and $T A Z$ between the two groups (Figure 5).

For the Wnt signaling pathway-related genes, the expressions of GBP, TCF, and LRP6 were downregulated in the Apatinib-treated group, while the expression of $D V l$ was upregulated, but there were no significant differences in the expressions of APC, AXIN, FZ, $\beta$-catenin (CTNNB1), C$M Y C, I L K, W N T, L E F$, and $C K 1$ between the two groups (Figure 6).

\section{Discussion}

Only a small fraction $(<1.0 \%)$ of tumor cells in tumor tissues have tumorigenic properties of self-renewal, unlimited proliferation, and multiple differentiation potentials similar 

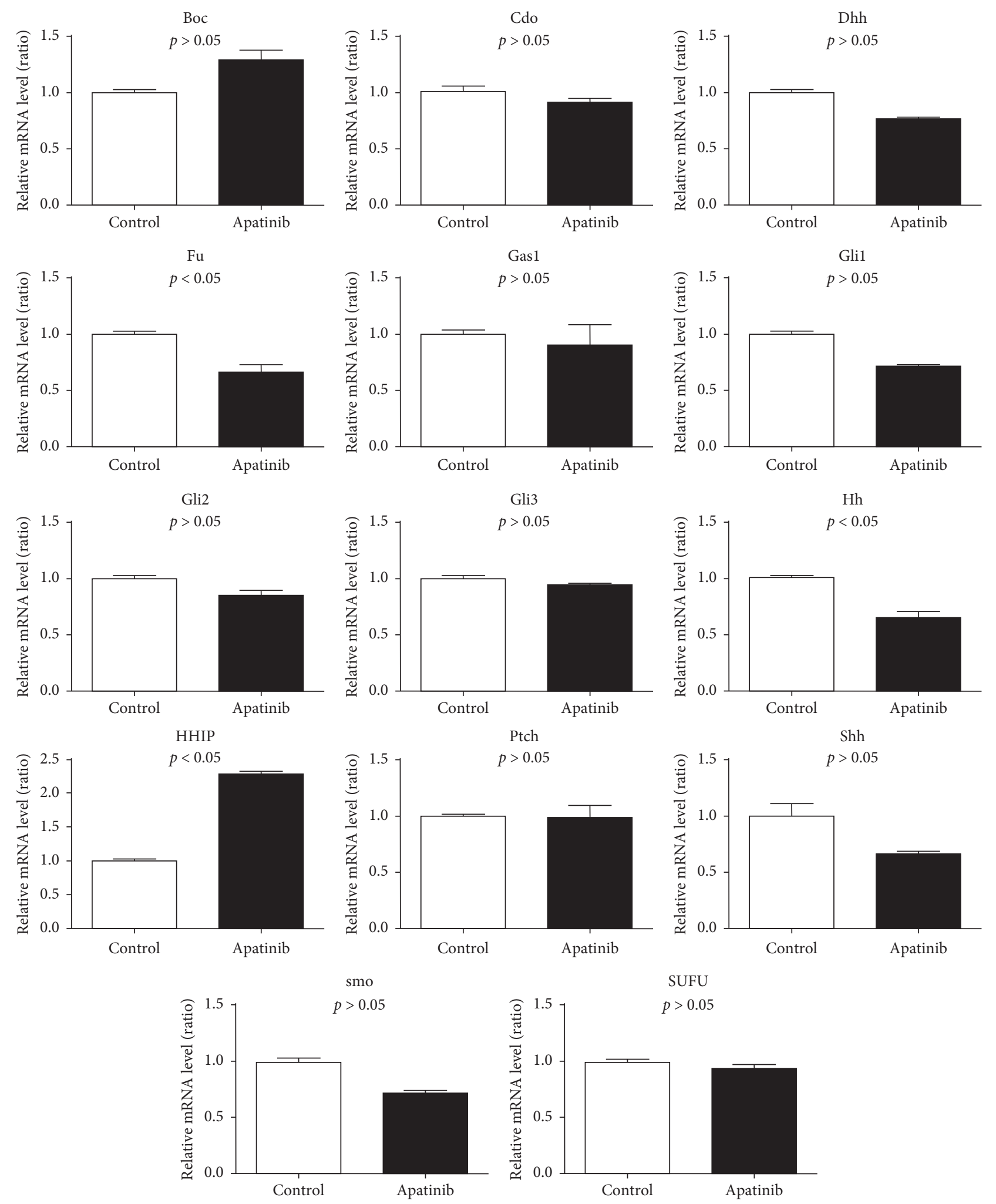

Figure 4: Effect of Apatinib on mRNA level of Hedgehog signaling pathway in lung cancer stem cells.

to those of normal stem cells. The formation and development of tumors are dominated by cancer stem cells (CSCs). A recent study analyzed gene expression profiles of several NSCLC primary cultures and cell lines and found that NANOG, NOTCH3, CD44, CDKN1A, SNAI1, and
ITGA6 were able to differentiate CSCs [10]. Lung cancer is one of the most common malignant tumors. With further studies carried out on lung cancer and LSCs, recent investigations have proven that TSCs also exist in lung cancer [11]. Several studies have shown that lung cancer stem cells 

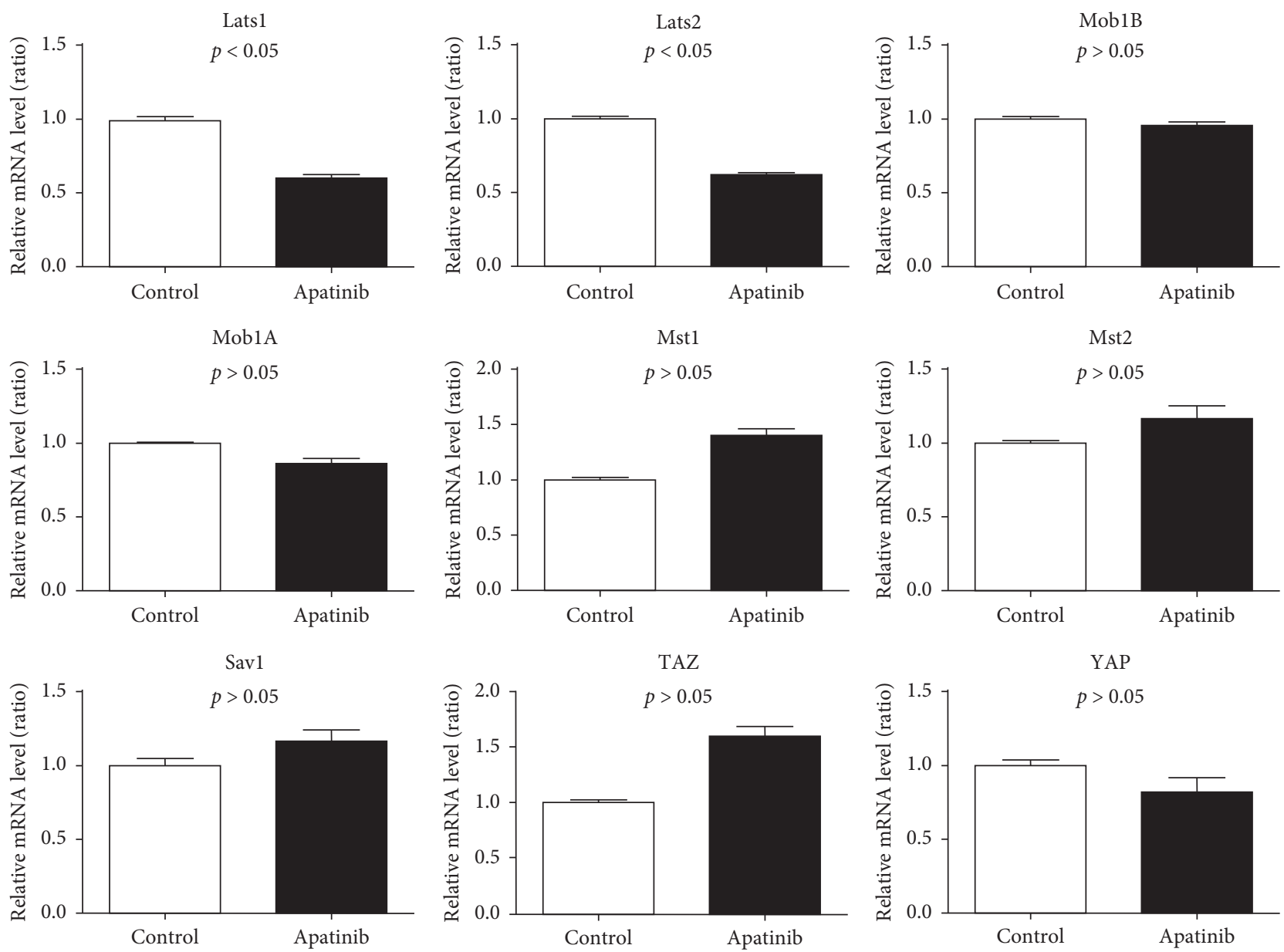

FIgURE 5: Effect of Apatinib on mRNA level of Hippo signaling pathway in lung cancer stem cells.

(LCSCs) play an important role in the occurrence, development, recurrence, and metastasis of this malignant disease. Besides, research has shown that LCSCs are highly resistant to radiotherapy and chemotherapy. This high drug resistance rate of lung cancer could also be closely related to LCSCs, whose mechanism mainly includes the following: (1) tumor stem cells have a strong self-repair ability and low level of reactive oxygen species (ROS), which can avoid the damage of DNA sequence through a DNA repairing mechanism and ensure cell activity; (2) the drug is pumped out of the cell with the help of an ATP-binding cassette transporter, which decreases the intracellular drug concentration; (3) lung cancer stem cells are in a state of retention (stage G0) and cannot be effectively divided and proliferated [12]. As a result, certain drugs acting on the cell proliferation phase cannot produce an effect, resulting in drug resistance. In this study, we found that Apatinib could inhibit the expressions of stemness biomarkers $A B C G 2$, CD24, ICAM-1, OCT4, and SOX2 in lung cancer.

Further analyses showed that the Hedgehog signaling pathway is activated significantly in both NSCLC and smallcell lung cancer. The Hedgehog signaling pathway, which is involved in nicotine activation of lung cancer, is the first step in the activation of lung cancer [13]. In this study, Apatinib inhibited the Hedgehog signaling pathway and downregulated the $S H H, H H$, and $F U$ of this signaling pathway. Therefore, Apatinib could be the first step to blocking the proliferation, invasion, and metastasis of NSCLC through the Hedgehog signaling pathway. The Hedgehog pathway is also involved in cancer resistance, such as cytotoxic chemotherapy, radiotherapy, and targeted therapy. Because the Hedgehog signaling pathway is related to ABC transporter, its inhibition is considered a chemosensitizer after chemotherapeutic drug resistance [14]. Della and other studies have shown that the Hedgehog signaling pathway is one of the mechanisms of drug resistance induced by EGFR inhibitors [15]. It could be suggested that Apatinib reverses chemoresistance by inhibiting the Hedgehog signaling pathway and, therefore, prolongs the PFS and OS of chemotherapy-resistant patients.

The activation of the Wnt signaling pathway is present in most lung cancer patients. Studies have shown that the Wnt signaling pathway has a significant effect on the occurrence, prognosis, and drug resistance of lung cancer $[16,17]$. Our investigation revealed that Apatinib reduces the expressions of TCF, GBP, and LRP6 in the Wnt signaling pathway significantly to inhibit the pathway, thus suppressing tumor and inhibiting the progression of lung cancer. The absence of any significant increase in the expressions of $A P C, A X I N$, WNT1, WNT2, WNT3, WNT5a, WNT7a, and $\beta$-catenin in 

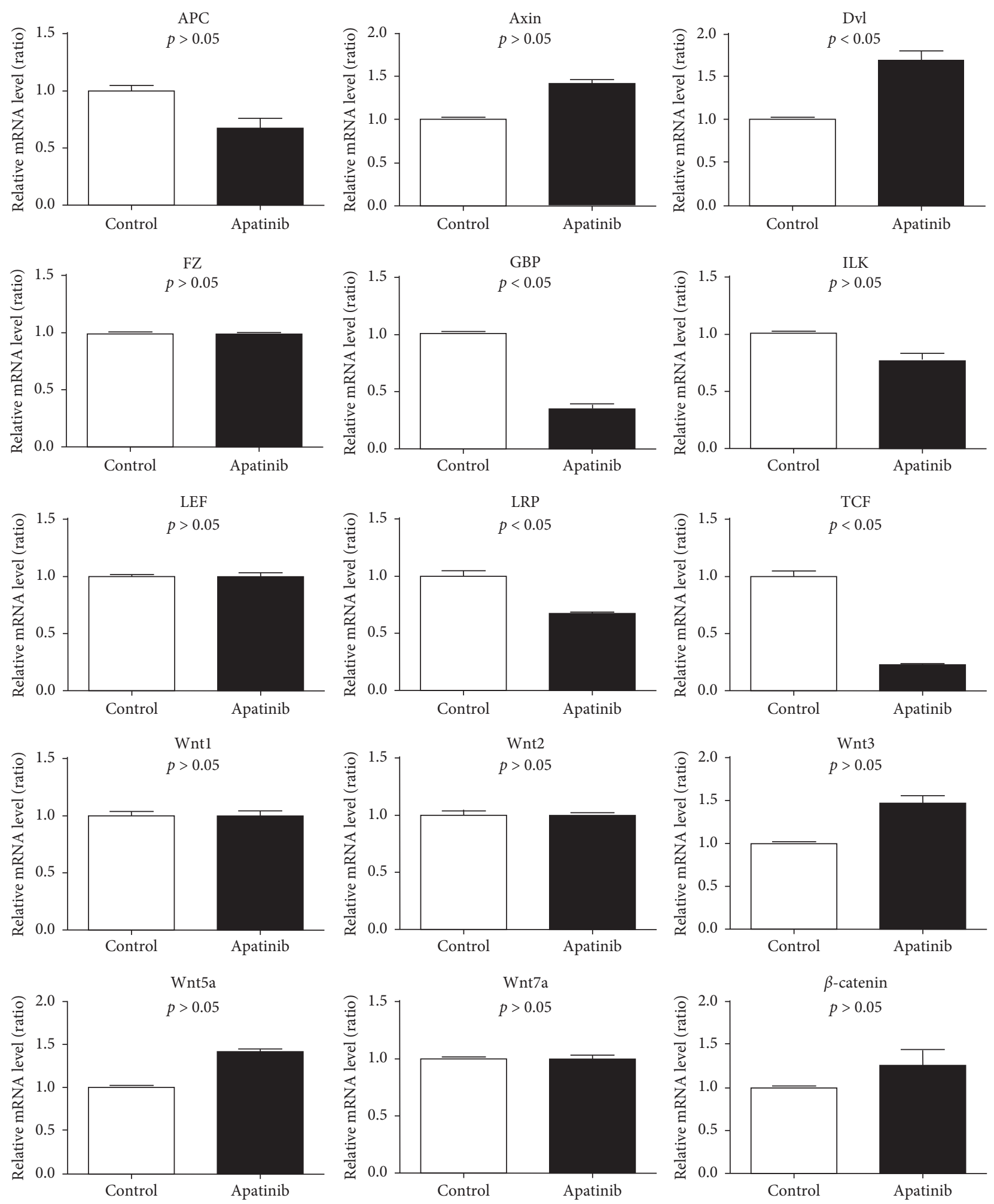

FIgURE 6: Effect of Apatinib on mRNA level of Wnt signaling pathway in lung cancer stem cells.

the Wnt signaling pathway within the Apatinib group could be due to the shorter drug-administration time; these target genes may be downregulated if treatment time is prolonged. Studies have demonstrated that the overexpression of WNT1 gene in the Wnt signaling pathway can induce the apoptotic resistance of cancer cells [18]. The expression of the multiple drug resistance gene (MDR1) is also closely related to the activation of the Wnt signaling pathway [19]. Therefore, we reasonably speculated that the mechanism by which Apatinib can improve the prognosis of chemotherapy-resistant 
patients significantly might be through the inhibition of the Wnt signaling pathway.

The Hippo signaling pathway, like the Hedgehog and Wnt signaling pathways, is a key member of the stemness pathway of lung cancer. The main components of the Hippo signaling pathway include $M S T 1 / 2, L A T S 1 / 2$, and YAP/TAZ. YAP and $T A Z$, located on the human chromosome 11q22, are overexpressed in NSCLC and regarded as oncogenes of lung cancer [20]. Although LATS1/2 was downregulated in our study, its upstream target, $M S T 1 / 2$, or downstream target, $Y A P / T A Z$, did not increase or decrease. Therefore, we speculated that Apatinib could lessen the expression of oncogene $Y A P / T A Z$, which in turn relieves the inhibition of the tumor suppressor genes $M S T 1 / 2$ and $L A T S 1 / 2$; they may also play roles at the same time in the process Apatinib works. However, the specific mechanism of Apatinib in the Hippo signaling pathway still needs further research to confirm.

In summary, our investigation found that Apatinib inhibited the growth of NSCLC subcutaneous xenografts by inhibiting the Hedgehog, Hippo, and Wnt pathways, which suppressed the stemness nature of lung cancer cells and then inhibited the progression of lung cancer. The Wnt, Hedgehog, and Hippo signaling pathways are potential targets for the diagnosis and treatment of NSCLC. By inhibiting these pathways, not only could tumor proliferation, invasion, and metastasis be repressed, but it is also expected to be a significant target for reversing drug resistance in NSCLC. This study provides a theoretical basis for the use of Apatinib in advanced NSCLC patients with multidrug resistance. The limitation of our study is that it was carried out only at the animal level. In-depth research to verify and explore the molecular mechanism at the human level is required in the future.

\section{Data Availability}

The data used to support the findings of this study are available from the corresponding author upon request.

\section{Conflicts of Interest}

The authors declare that they have no conflicts of interest.

\section{Authors' Contributions}

Bin Yang and Yan Wang contributed equally to this work.

\section{Acknowledgments}

This study was supported by the Research Project of Hubei Provincial Science and Technology Department (2018CFC846) and Henry CSCO-Cancer Research Fund (Y-HR2017-033).

\section{References}

[1] J. Ferlay, I. Soerjomataram, R. Dikshit et al., "Cancer incidence and mortality worldwide: sources, methods and major patterns in GLOBOCAN 2012," International Journal of Cancer, vol. 136, no. 5, pp. E359-E386, 2015.
[2] American Cancer Society, Cancer Facts \& Figures 2015, American Cancer Society, Atlanta, GA, USA, 2015.

[3] F. R. Hirsch, G. V. Scagliotti, J. L. Mulshine et al., "Lung cancer: current therapies and new targeted treatments," The Lancet, vol. 389, no. 10066, pp. 299-311, 2017.

[4] P. Kou, Y. Zhang, W. Shao et al., "Significant efficacy and well safety of apatinib in an advanced liver cancer patient: a case report and literature review," Oncotarget, vol. 8, no. 12, pp. 20510-20515, 2017.

[5] H. F. Dvorak, "Tumor stroma, tumor blood vessels, and antiangiogenesis therapy," The Cancer Journal, vol. 21, no. 4, pp. 237-243, 2015.

[6] D. H. Kong, M. Kim, J. Jang, H.-J. Na, and S. Lee, "A review of anti-angiogenic targets for monoclonal antibody cancer therapy," International Journal of Molecular Sciences, vol. 18, no. 8, p. 1786, 2017.

[7] X. Wang, W. Zhang, W. Du et al., "Efficacy and survival analysis of apatinib in patients with advanced nonsquamous non-small cell lung cancer after failure of first-line treatment," Zhongguo Fei Ai Za Zhi/Chinese Journal of Lung Cancer, vol. 20, no. 11, pp. 761-768, 2017.

[8] G. Ahmad and M. M. Amiji, "Cancer stem cell-targeted therapeutics and delivery strategies," Expert Opinion on Drug Delivery, vol. 14, no. 8, pp. 997-1008, 2017.

[9] K. Eun, S. W. Ham, and H. Kim, "Cancer stem cell heterogeneity: origin and new perspectives on CSC targeting," $B M B$ Reports, vol. 50, no. 3, pp. 117-125, 2017.

[10] A. Herreros-Pomares, J. D. de-Maya-Girones, S. CalabuigFariñas et al., "Lung tumorspheres reveal cancer stem cell-like properties and a score with prognostic impact in resected non-small-cell lung cancer," Cell Death \& Disease, vol. 10, no. 9 , p. $660,2019$.

[11] S. Holmboe, P. L. Hansen, H. Thisgaard et al., "Evaluation of somatostatin and nucleolin receptors for therapeutic delivery in non-small cell lung cancer stem cells applying the somatostatinanalog DOTATATE and the nucleolin-targeting aptamer AS1411," PLoS One, vol. 12, no. 5, Article ID e0178286, 2017.

[12] R. Suresh, S. Ali, A. Ahmad, P. A. Philip, and F. H. Sarkar, "The role of cancer stem cells in recurrent and drug-resistant lung cancer," Lung Cancer and Personalized Medicine: Novel Therapies and Clinical Management, Springer, Cham, Switzerland, pp. 57-74, 2016.

[13] Y. Guo, G. Shi, H. Wan, and M. Zhou, "Hedgehog signaling regulates the expression levels of inflammatory mediators in cigarette-induced airway inflammation," Molecular Medicine Reports, vol. 17, no. 6, pp. 8557-8563, 2018.

[14] J. Sims-Mourtada, J. G. Izzo, J. Ajani, and K. S. C. Chao, "Sonic Hedgehog promotes multiple drug resistance by regulation of drug transport," Oncogene, vol. 26, no. 38, pp. 5674-5679, 2007.

[15] C. M. Della Corte, U. Malapelle, E. Vigliar et al., "Efficacy of continuous EGFR-inhibition and role of Hedgehog in EGFR acquired resistance in human lung cancer cells with activating mutation of EGFR," Oncotarget, vol. 8, no. 14, pp. 2302023032, 2017.

[16] T. Zhan, N. Rindtorff, and M. Boutros, "Wnt signaling in cancer," Oncogene, vol. 36, no. 11, pp. 1461-1473, 2017.

[17] D. J. Stewart, "Wnt signaling pathway in non-small cell lung cancer," JNCI: Journal of the National Cancer Institute, vol. 106, no. 1, 2014.

[18] S. Chen, D. C. Guttridge, Z. You et al., "WNT-1 signaling inhibits apoptosis by activating $\beta$-catenin/T cell factor-mediated transcription," Journal of Cell Biology, vol. 152, no. 1, pp. 87-96, 2001. 
[19] Z. Xia, M. Guo, H. Liu et al., "CBP-dependent Wnt/ $\beta$-catenin signaling is crucial in regulation of MDR1 transcription," Current Cancer Drug Targets, vol. 15, no. 6, pp. 519-532, 2015.

[20] F. Lo Sardo, S. Strano, and G. Blandino, "YAP and TAZ in lung cancer: oncogenic role and clinical targeting," Cancers, vol. 10, no. 5, p. 137, 2018. 